\title{
Continuous Close-Proximity RSSI-based Tracking in Wireless Sensor Networks
}

\author{
Gaddi Blumrosen*§, Bracha Hod ${ }^{* \S}$, Tal Anker*, Danny Dolev* and Boris Rubinsky*† \\ ${ }^{*}$ School of Computer Science and Engineering, The Hebrew University of Jerusalem, Israel \\ Email: \{gaddi, hodb, anker, dolev, rubinsky\}@ cs.huji.ac.il \\ ${ }^{\dagger}$ Department of Mechanical Engineering, University of California at Berkeley, Berkeley, CA
}

\begin{abstract}
In this paper we develop a continuous high-precision tracking system based on Received Signal Strength Indicator (RSSI) measurements for small ranges. The proposed system uses minimal number of sensor nodes with RSSI capabilities to track a moving object in close-proximity and high transmission rate. The close-proximity enables conversion of RSSI measurements to range estimates and the high transmission rate enables continuous tracking of the moving object. The RSSI-based tracking system includes calibration, range estimation, location estimation and refinement. We use advanced statistical and signal processing methods to mitigate channel distortion and packet loss. The system is evaluated in indoor settings and achieves tracking resolution of few centimeters. Therefore, it becomes the motion trackers of notice in many applications.
\end{abstract}

\section{INTRODUCTION}

Different motion tracking technologies are used in medicine, sport and military applications to assess motion patterns, e.g., gait analysis for patients with neurological disorders, such as Parkinson. The most common motion tracking device is a motion sensor that consists of accelerometers and gyroscopes to measure the accelerations and angular velocities, respectively.

The advantages of motion sensors over other techniques are in terms of cost and ease-of-use. However, the motion sensors suffer from an increased drift over time, which affects the accuracy of the output and requires continuous calibration. Wearable Body Sensor Networks (BSN) [1] mounted with motion tracking devices are recently used for motion analysis. These sensors exploit the BSN computation power and the wireless capabilities for efficient continuous motion tracking in any environment. Every BSN node supports also the Received Signal Strength Indicator (RSSI), which is an alternative tracking mechanism.

RSSI is a measurement of the signal power on a radio link [2]. It has been extensively used as one of the ranging techniques in Wireless Sensor Networks (WSNs) due to its simplicity, low power consumption and economical price. Existing RSSI-based tracking systems are affected by the channel conditions and provide a resolution in order of meters, which is not adequate for precise motion tracking needed for medical applications.

In this paper, we suggest using RSSI-based tracking, for medical application. The motivation of this paper is to show

\footnotetext{
$\S$ Contributed equally to this work.

This paper was accepted for publication in the proceedings of 2010 International Conference on Body Sensor Networks.
}

that RSSI is a valid tool for continuous position estimation and tracking of a proximate object with high transmission rate. In close proximity and Line Of Sight (LOS) conditions, with accurate calibration, it is possible to increase the accuracy of distance estimation to scale of centimeters. With high transmission rate we can further exploit the diversity of consecutive RSSI measurements, which refer to proximate location of the moving object. Advanced processing techniques we developed enable exploiting the diversity in RSSI samples to mitigate over channel distortion and packet loss. The proposed method uses fewer sensor nodes than other solutions, and therefore provides more economical solution. Experiments show a motion tracking in resolution of few centimeters.

The paper is organized as follows. Section II provides some background information and reviews related work in this area. Section III gives description of the system model and the problem formulation. The data processing algorithms we use are described in Section IV] Section V provides the experiment setup and Section VI describes the experiment results. Conclusion and discussion about future work are presented in Section VII

\section{RELATED WORK}

Conventional RSSI-based location and tracking schemes, e.g., [3], [4], [5], [6], estimate the range between pairs of nodes using known channel model characteristics or some calibration methods. Then, apply methods such as triangulation, trilateration, or statistical inference like maximum likelihood or Bayesian estimation to obtain the location [7].

Common range estimation techniques use the path-loss model. RSSI characteristics according to this model are considered in [8], [9] and [10]. The path-loss model is statistical model and cannot overcome fast fading effect, reflections from walls, shadowing and non-isotropic antenna gains. A calibration is often used to either find channel model parameters or to produce a conversion table between RSSI measurements and distances, e.g., [11], [12], [13].

RSSI-based range estimation methods have been studied in several works, e.g., [14]. The range estimation is not accurate as it is sensitive to small variations in channel and is usually in scale of meters. In proximate environment, with accurate calibration and LOS conditions, [15] obtained approximation error of up to $10 \mathrm{~cm}$ using raw RSSI measurements without further processing. The works described in [16] and [17] 
provide more advanced RSSI processing methods, such as histogramic analysis and statistical filters that improved range accuracy.

RSSI-based tracking exploit diversity of measurements and motion models. Classical approaches for tracking are based on Kalman filters [18] or more general Bayesian filters like particle filters [19].

\section{SYSTEM MODEL}

\section{A. System Description}

The basic system consists of a single mobile node with a location of $\left(x_{0}, y_{0}, z_{0}\right)$ in Cartesian coordinates and $N$ static nodes, referred to as anchor nodes, placed at $\left(x_{1}, y_{1}, z_{1}\right),\left(x_{2}, y_{2}, z_{2}\right), . .,\left(x_{N}, y_{N}, z_{N}\right)$, respectively. The goal of our work is to continuously estimate the mobile node's location $\left(x_{0}^{t}, y_{0}^{t}, z_{0}^{t}\right)$ at any given time $t$. The mobile node transmits a data packet with a known transmission power to the anchor nodes every $T$ ms. The anchor nodes, located in the transmission range of the mobile node, calculate the received power values $\operatorname{Pr}_{1}^{t}, \operatorname{Pr}_{2}^{t}, \ldots, \operatorname{Pr}_{N}^{t}$. Each transmitted packet is labeled with a time stamp, to recover possible packets loss. No synchronization is assumed among the nodes. The received signal power using channel pass-loss model for anchor node $i$ at time $t$ is:

$$
P r_{i}^{t}=P t+A-q 10 \log _{10} d_{i}^{t}+\alpha^{t},
$$

where $d_{i}^{t}$ is the distance between anchor node $i$ and the mobile node, $A$ is a constant power offset, which is determined by several factors, like receiver and transmitter antenna gains and transmitter wave length [20], $q$ is the channel exponent which vary between 2 (free space) and 4 (indoor with many scatterers), and $\alpha^{t}$ is a Gaussian distributed random variable with zero mean and standard deviation $\sigma$ that accounts for the random effect of shadowing.

\section{B. Problem Formulation}

Denote the power measurement matrix of $N$ anchor nodes over $M$ time units by $P r$ :

$$
\mathbf{P r}=\left(\begin{array}{cccc}
\operatorname{Pr}_{1}^{1} & \operatorname{Pr}_{1}^{2} & \ldots & \operatorname{Pr}_{1}^{M} \\
\operatorname{Pr}_{2}^{1} & \operatorname{Pr}_{2}^{2} & \ldots & \operatorname{Pr}_{2}^{M} \\
\vdots & \vdots & \vdots & \vdots \\
\operatorname{Pr}_{N}^{1} & \operatorname{Pr}_{N}^{2} & \ldots & \operatorname{Pr}_{N}^{M}
\end{array}\right) .
$$

To track the mobile node, we need to continuously estimate, using the set of $N$ power measurements, the location of the mobile node. The Minimum Mean Square Error (MMSE) optimal transformation of the measurement matrix $\mathrm{Pr}$ can be obtained by solving the following criterion:

$$
\hat{f}=\operatorname{argmin}_{f} \mathbb{E}\left(X_{0}-f(P r)\right)^{2} \text { s.t. }\left|X_{0}^{t+1}-X_{0}^{t}\right|<\delta,
$$

where $X_{0}$ consists of $M$ consecutive coordinates of the mobile node, $f$ is a transformation of the power measurements to location, $\mathbb{E}[\cdot]$ is the expected value over all stochastic sources, and $\delta$ is a bound on the difference between consecutive location estimations, which is a function of transmission rate and mobile node velocity. With high RSSI transmission rate or low mobile node velocity, consecutive RSSI measurements imply proximate locations.

The problem is neither linear and nor convex [21], thus the criterion in (2) can only be solved numerically. Furthermore, an optimal transformation requires accurate statistical knowledge [14], which is not always available. Since the mobile node moves during observation time, the channel is not stationary and new frequent update of the transformation is needed for accurate approximation.

\section{DATA PROCESSING AND ANALYSIS}

We assume that the errors in anchor RSSI measurements are independent. As a result, we can separate the tracking solution to (2) into two phases. In first phase we estimate the distance (range) between the mobile node and each anchor node. In the second phase we integrate the entire information to obtain MMSE optimal location estimation. The solution has the following four stages: (a) pre-processing of the RSSI measurements to obtain the received power. This stage includes conversion of the RSSI measurements to power measurements, interpolation of missing samples and filtering out the channel noise; (b) range estimation between the mobile node and each anchor node according to the power measurements and calibration; (c) combination of the information from all the nodes and the MMSE estimation of the mobile node's location; and (d) filtering out estimation errors with statistical methods.

\section{A. Pre-Processing of RSSI Measurements}

The 8-bit RSSI measurements are converted to power, as described in [22]. As there might be missing packets and our solution is based on continuous measurements, we approximate the missing packets by linear interpolation. To exclude the noise components in (1), we filter the interpolated data for each anchor node with a low pass filter:

$$
\tilde{\operatorname{Pr}_{i}^{t}}=\operatorname{Pr}_{i}^{t} * h
$$

where * denotes the convolution operation and $h$ is a lowpass filter that smoothes the additive noise and eliminates the fast-fading.

\section{B. Range Estimation}

A continuous estimation of the distance between the mobile node and an anchor node $i$ can be derived analytically from the filtered received power according to [1]:

$$
\tilde{d}_{i}^{t}=10^{\frac{P t+A-\tilde{P r} r_{i}^{t}}{10 q}} \text {. }
$$

This range approximation requires a-priory knowledge of channel parameters, channel exponent value and receive and transmit antenna gains, which determine the exponent offset. Using common channel exponent for indoor in range of $2-$ 4 will not provide accurate results and will not compensate specific channel condition like shadowing.

Calibration is necessary to reflect the specific medium. A common calibration approach in [9], uses one reference point in the medium with a known measured received power. 
This calibration approach compensates for the bias induced by the receive and transmit antennas gains, but still uses the inaccurate channel exponent. Another calibration approach is to derive both channel offset and exponent for a predetermined distance before operation, like in [7]. Alternative calibration method, known as fingerprinting, creates a database of RSSI values as a function of distances. We use a variant of the fingerprinting method. We measure the RSSI values at different distances. These measurements form a curve of distance as a function of RSSI measurements. Unlike fingerprinting method, we further use a polynomial fitting for the curve to exclude the effect of noisy measurements. We store the result in a mapping table. We use the RSSI value to fetch the closest distances and use linear interpolation to improve accuracy. This calibration approach is accurate in stationary channel but is not accurate when the channel varies.

\section{Location Estimation}

Denote by $\tilde{D}$ the matrix of approximated distances calculated above:

$$
\tilde{\mathbf{D}}=\left(\begin{array}{cccc}
\tilde{d}_{1}^{1} & \tilde{d}_{1}^{2} & \ldots & \tilde{d}_{1}^{M} \\
\tilde{d}_{2}^{1} & \tilde{d}_{2}^{2} & \ldots & \tilde{d}_{2}^{M} \\
\vdots & \vdots & \vdots & \vdots \\
\tilde{d}_{N}^{1} & \tilde{d}_{N}^{2} & \ldots & \tilde{d}_{N}^{M}
\end{array}\right)
$$

The following criteria can estimate the mobile node's location:

$$
\hat{g}=\operatorname{argmin}_{g} \mathbb{E}\left(X_{0}-g(\tilde{D})\right)^{2} \text { s.t. }\left|X_{0}^{t+1}-X_{0} t\right|<\delta .
$$

There are several methods for solving (5). The most common one is trilateration. Trilateration is a positioning technique, [23], which estimates the mobile node's location by intersection of the circles, each centered on the anchor node position, with a radius equals to the estimated distance between the mobile node and the anchor node. $N=p+1$ anchor nodes are required for localization in $p$ dimensional space. The estimated location is defined by the center of the region formed by the intersection of the circles. Another approach [24] utilizes only $N=p$ anchor nodes and estimates the location by one of the intersection points. It records several intersection points in consecutive times and estimates the intersection location by the closest distance.

We choose a variant of [24] to estimate the mobile node's location using the Maximum A Posteriori (MAP) criterion. Assuming that the range estimations have the same statistical distribution and the mobile node location has Gaussian distribution, the MAP criterion coincides with the MMSE criterion, [25]. The solution is composed of the following steps: 1) deriving intersection of the circles formed by the estimated distance for each anchor node, 2) choosing the intersection that minimizes the MAP criterion.

1) Deriving Circles' Intersections Points: To estimate the mobile node's location we use the intersection of the circles described in previous section. For 2-D with two anchor nodes, the circles formed by the distance estimation are:

$$
\left(x-r_{x}\right)^{2}+y^{2}=\left(\hat{d}_{x}^{t}\right)^{2}
$$

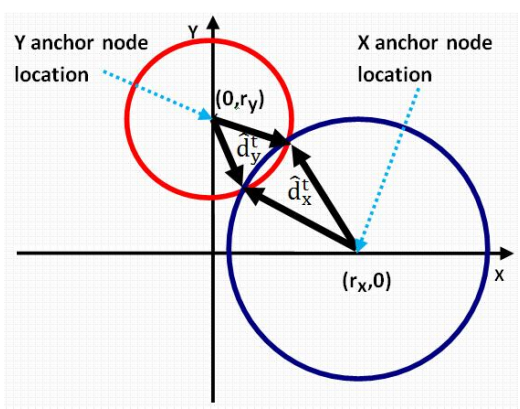

Fig. 1. Intersection points of two anchor nodes' circles. The two circles are centered on the anchor nodes' positions with radiuses equal to the estimated distance between the mobile node and the anchor nodes.

$$
x^{2}+\left(y-r_{y}\right)^{2}=\left(\hat{d}_{y}^{t}\right)^{2},
$$

where $r_{x}, r_{y}$ are the anchor nodes locations in the $\mathrm{x}$ and $\mathrm{y}$ axis. The intersections of the two circles are two points that one of them indicates the location of the mobile node, as illustrated in Figure 1 .

2) Choosing the Optimal Intersection Points: We want to choose the intersection points that are likely to be the mobile node location and minimize (5) in MMSE sense. We define a state as one intersection point. In 2-D with two anchor nodes, typically, there are two states, $S_{1}^{t}$ and $S_{2}^{t}$, for each intersection point. We use a trellis diagram that consists of all states in window of $W$ samples. A path in the diagram is a transition between states at consecutive discrete time intervals. Each possible transition represents a possible motion of the sensor from one position to another. To minimize the criteria in (5) we need to find the most likely path in the trellis diagram. Each legal transition between states can be defined as a branch with a branch metric $S$, which is a function of the distance between consecutive states. We use a branch metric that reflects the continuity constraint in (5). A branch metric can be based on proximity of consecutive samples and is given by:

$$
B M_{d}^{t}=\left\|d\left(S_{i}^{t+1}\right)-d\left(S_{j}^{t}\right)\right\|,
$$

where $\|\cdot\|$ is Euclidian norm. Another branch metric can be based on continuity of mobile node's motion and is given by:

$$
B M_{v}^{t}=\left\|v\left(S_{i}^{t+1}\right)-v\left(S_{j}^{t+1}\right)\right\| .
$$

The velocity $v$ can be either linear or angular in polar coordinates. A path metric is the sum of the branch metrics for a window length of $W$ samples:

$$
P M^{t}=\sum_{t^{\prime}=t-W}^{t} B M^{t^{\prime}} .
$$

$W$ is also called the constraint length, $W<<M$.

The MAP criterion chooses the minimum path metric out of all the possible paths. A more efficient algorithm can use the Maximum Likelihood (ML) criterion, which can be implemented by Viterbi algorithm [26]. If we assume that the distance distribution is i.i.d, both MAP and ML solutions minimize the error criterion in (5] [25]. Figure 2 illustrates a selection of the intersection point that estimates the mobile node's location. 


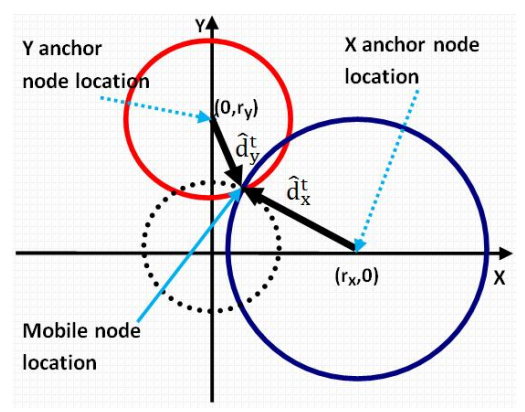

Fig. 2. Selection of the intersection point that is related to the mobile node's location.

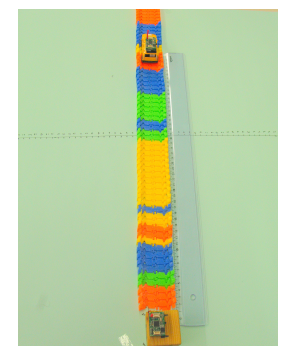

(a) Calibration stage in which we form a mapping table between power measurements and distances.

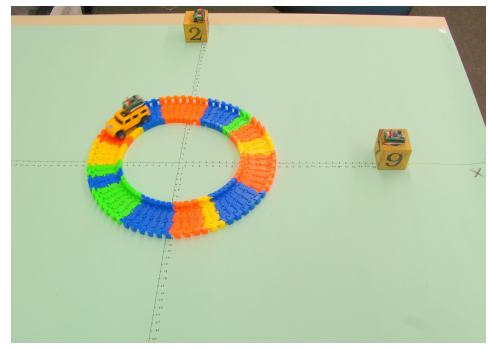

(b) Experiments using circular trail. The two anchor nodes calculate the RSSI from the mobile node transmissions and transmit the results to a computer for further processing.
Fig. 3. Experiment setup.

\section{Post Processing}

We use additional filtering to exclude decisions that are not likely and to smooth the results. First, we apply on the approximated distances a median filter [27]. The filter extracts approximations that are different in relation with the standard deviation from the mean value and are likely to be errors. We then use a linear interpolation value instead of the noisy distances and then we use a low-pass filter on the results.

\section{EXPERIMENT SETUP}

The experimental setup includes a trail with a toy car, two anchor nodes, one mobile node, one base station and a computer. The mobile node is attached to the toy car, which moves on a circular plastic trail that is wider than the car itself. RSSI measurements were sent to the computer through the base station for analysis.

The two anchor nodes and the mobile node are BSN nodes. A BSN node includes a processing unit (TI MSP430), transceivers for the wireless communication (Chipcon CC2420) [22] and a monopole antenna. An additional isotropic dipole antenna with length of $4 \mathrm{~cm}$ was added to increase the transmission range. The CC2420 offers a mechanism for selecting the transmission output power of the radio in range from $-25 \mathrm{dBm}$ to $0 \mathrm{dBm}$. The actual transmission power we use is $-7 \mathrm{dBm}$ (Power level 15). The CC2420 has a built-in RSSI providing a digital value in range

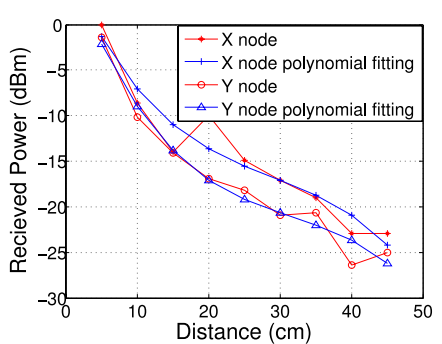

Fig. 4. Results of the calibration between the two anchor nodes and the mobile node. The difference between the two anchor nodes can be explained either by different antenna gains or by varying channel conditions.

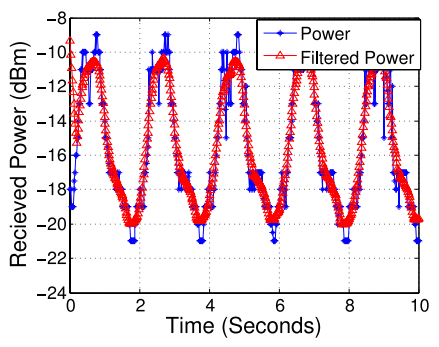

(a) Node $\mathrm{x}$.

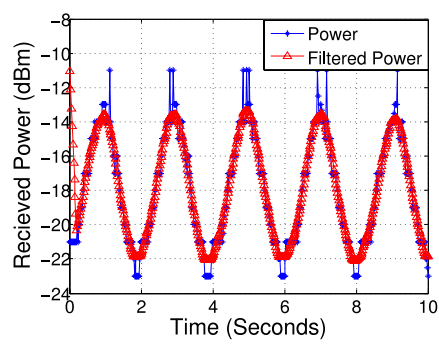

(b) Node y.
Fig. 5. Received powers of the two anchor nodes with and without filtering.

of -127 to $128 \mathrm{dBm}$. The RSSI value is always averaged over 8 symbol periods $(128 \mathrm{~ms})$. The conversion of the RSSI measurements to the received power is done by an addition of $-45 \mathrm{dBm}$.

The experiments were performed in indoor environment, with no metal reflectors in a range of 1 meter from the nodes so they were nearly in line-of-sight conditions. The anchor nodes are located in the $x$ and $y$ axis, in coordinates of $(30 ; 0)$ and $(0 ; 30)$ centimeters respectively. Figure 3 shows the experiment setup.

At first phase we performed calibration for 9 different distances between the mobile node and each anchor node. Then, we examined circular trail with 4 sets of radiuses: 10 , 12,14 and $16 \mathrm{~cm}$. The toy car, traveled over the trail, moved with a constant velocity of $0.33 \mathrm{~m} / \mathrm{s}$ and the sensor node attached to it transmits a data packet with time stamp every $20 \mathrm{~ms}$. Each anchor node computed the received power as in the calibration phase and transmitted it to the processing unit. The overall delay is composed mainly from the transmission delay and the constraint length.

\section{EXPERIMENT RESULTS}

The calibration between the mobile node and each of the anchor nodes was performed with 9 different distances from $5 \mathrm{~cm}$ to $45 \mathrm{~cm}$ in ascending order. The anchor node received packets transmitted from the mobile node with a known power level, calculated the receive power level and sent it to the processing unit for analysis. In the processing unit, we used a degree 3 polynomial fitting and stored the results in a mapping table. Figure 4 shows the calibration points and its log fitting for 2-D. 


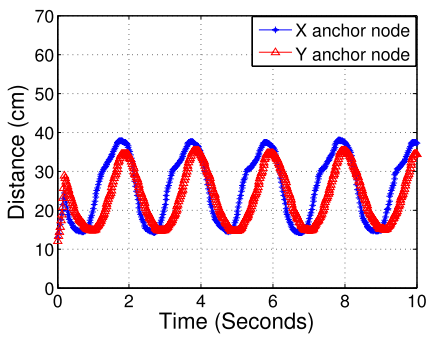

(a) Experiment distance estimation.

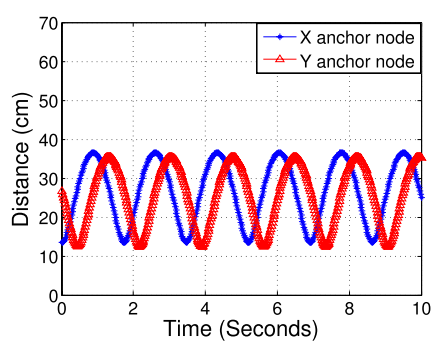

(b) Theoretical distance estimation.
Fig. 6. Distance Approximation between the mobile node and the two anchor nodes. The Theoretical distance estimation is for a circular movement.

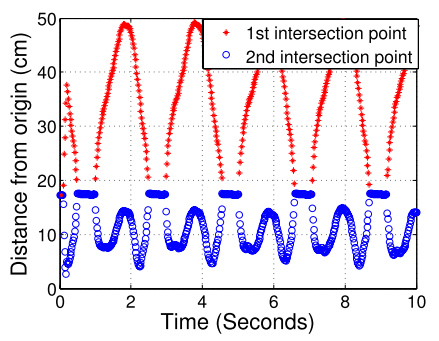

(a) Experiment distance.

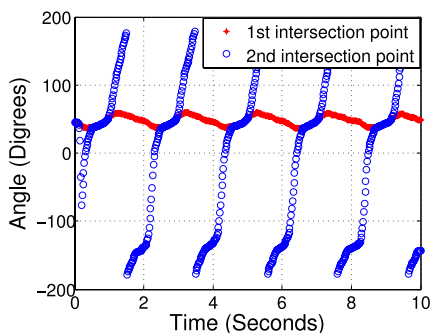

(c) Experiment angles.

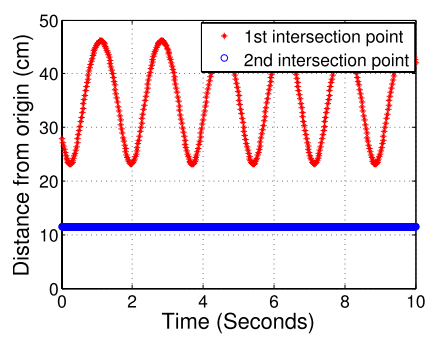

(b) Theoretical distance.

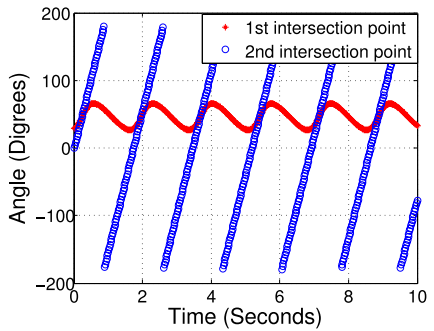

(d) Theoretical angles.
Fig. 7. The First and Second intersection points in polar coordinates.

Figure 5 presents the received power of the two anchor nodes, before and after low-pass filtering as described in Eq. (3), for a circle with radius of $12 \mathrm{~cm}$. We used the mapping table produced from the calibration stage to convert the power measurements to distances. We used linear interpolation between two power measurements to obtain the distance.

The distance approximation between the mobile node and the two anchor nodes in circular movement is shown in Figure 6 A phase shift of a quarter of a cycle (90 degrees) between $x$ and $y$ nodes can be noticed in the distance approximation as in theory. The amplitude of the $y$ 'th anchor node is $2 \mathrm{~cm}$ lower than the $x$ 'th approximation. The difference can be explained either by inaccurate calibration process or by non ideal isotropic antennas we used in our experiment.

We found the intersection points of the circles, which are formed by the distances between the anchor nodes and the mobile nodes by solving Eq. (6). Figure 7 describes the

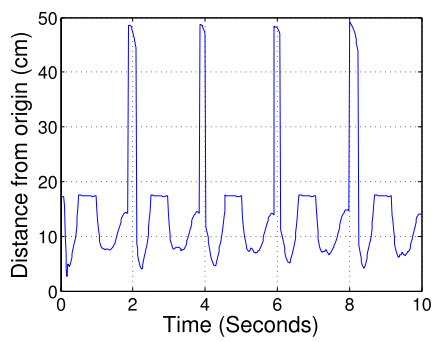

(a) Distance before processing.

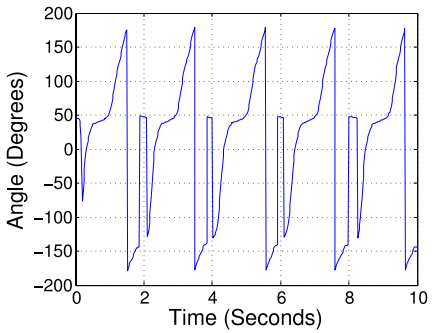

(c) Angle before processing.

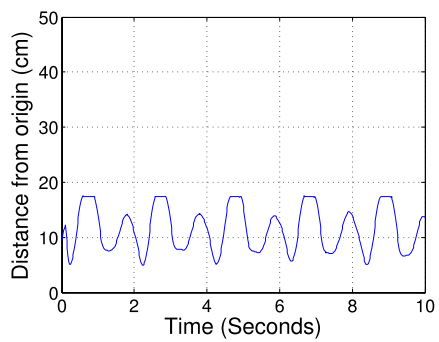

(b) Distance after processing.

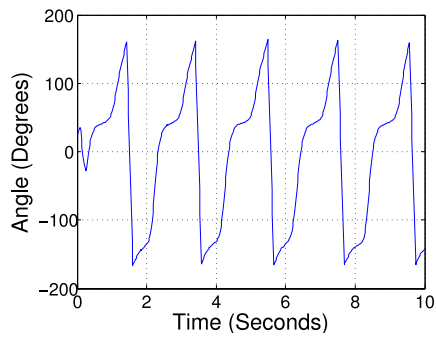

(d) Angle after processing.
Fig. 8. The approximated distance from origin and the angle of the mobile node, before and after the post-processing stage.

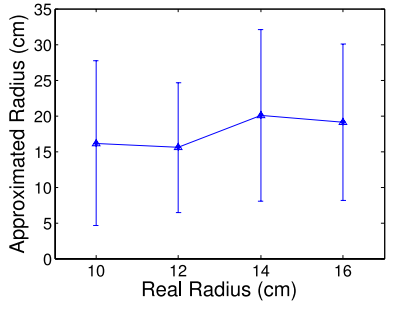

(a) Before post-processing.

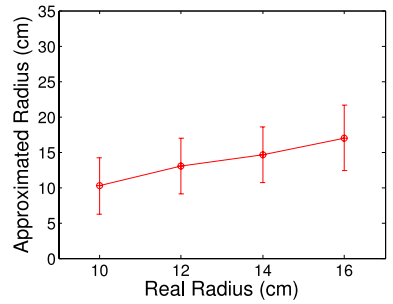

(b) After post-processing
Fig. 9. The error mean and standard deviation of the mobile location. The figure in the left shows noisy results with average error of $4.7 \mathrm{~cm}$ and standard deviation of $10 \mathrm{~cm}$. The figure in the right shows relatively accurate results after post processing with average error of $0.7 \mathrm{~cm}$ and standard deviation of $4 \mathrm{~cm}$.

intersection of the two circles in polar coordinates. The mobile node's location is estimated according to Eq. (7)-(9).

We then post process the location estimation as described in Section IV-C1 Figure 8(a) and Figure 8(c) show the approximated distance and the angle of the mobile node from the origin before the post-processing stage. Before filtering we can see a periodic burst of estimation errors in both radius and angel approximations. An estimation error can be explained by choosing the wrong intersection, which does not indicate the correct mobile node's location. The burst of errors means that a consecutive series of the wrong intersections were chosen. In circular movement, the wrong intersections are proximate almost as much as the true mobile node location. This difference in proximity is the noise tolerance of the 
algorithm. Burst errors can be a result of a distortion in system, either by non ideal isotropic antennas or by packet loss due to communication failure and imperfect reconstruction. With higher constraint length we include more statistics in the decision and reduce the probability of error, but increase the total delay. Figure 8(b) and Figure $8(\mathrm{~d})$ show the approximated distance and the angle of the mobile node from the origin after the post-processing stage. It can be noticed that the error bursts were filtered successfully.

The error mean and standard deviation of the approximated location for the different circular trails before the post processing were $4.7 \mathrm{~cm}$ and $10 \mathrm{~cm}$, respectively. After the post processing stage, the error mean and standard deviation were reduced to $0.7 \mathrm{~cm}$ and $4 \mathrm{~cm}$, respectively. Though our experiment is limited to circular paths, the standard deviation gives indication for the location approximation error in the general case of movement as we used low constraint length and relatively high sampling rate. Consequently, it seems that in a general tracking we will be able to achieve accuracy of $4 \mathrm{~cm}$ in location estimation. The mean error and standard deviation for all trails before and after post processing are shown in Figure 9 .

\section{CONCLUSIONS AND FUturE WORK}

In this paper we suggest a new real-time RSSI-based tracking system for continuous tracking in close-proximity of up to 1 meter, using high transmission rate. We use an advanced calibration method combined with RSSI-based ranging and MAP location estimation. We further use advanced filtering techniques to mitigate over channel distortion and packet loss. Our method assumes that consecutive RSSI samples refer to the same location, and the processing of the results was made according to this assumption. Therefore, although the experiments were taken over a circular path, the results are relevant for other motion patterns as well. We demonstrate our system using a mobile node, moving on a circular path, and two anchor nodes located at proximate distance. We used an isotropic antenna for simplicity. The experiment shows a distance estimation error for the radius of only $0.7 \mathrm{~cm}$ with standard deviation of $4 \mathrm{~cm}$ for a single measurement. The standard deviation gives indication of the location approximation error in the general case of movement as we used low constraint length and relatively high sampling rate. The proposed system suffers from inaccurate calibration and channel distortion over time. Auto-calibration during activation can exclude the need for calibration in advance and mitigate channel distortion in non-LOS conditions. The suggested system can provide in the future a robust and economical solution for tracking and can be considered as an alternative to inertial motion trackers for medical applications.

\section{REFERENCES}

[1] G.Z. Yang and M. Yacoub. Body sensor networks. Springer-Verlag New York Inc, 2006.

[2] T. Rappaport. Wireless communications: principles and practice. Prentice Hall PTR Upper Saddle River, NJ, USA, 2001.
[3] M. Sichitiu and V. Ramadurai. Localization of wireless sensor networks with a mobile beacon. Proceedings of MASS, pages 174-183, 2004.

[4] M. Bertinato, G. Ortolan, F. Maran, R. Marcon, A. Marcassa, F. Zanella, M. Zambotto, L. Schenato, and A. Cenedese. RF Localization and tracking of mobile nodes in Wireless Sensors Networks: Architectures, Algorithms and Experiments. University of Padua, Italy, 2007.

[5] W.Y. Chung. Enhanced RSSI-Based Real-Time User Location Tracking System for Indoor and Outdoor Environments. In Proceedings of the 2007 International Conference on Convergence Information Technology, pages 1213-1218. IEEE Computer Society Washington, DC, USA, 2007.

[6] H.J. Lee, M. Wicke, B. Kusy, and L. Guibas. Localization of mobile users using trajectory matching. In Proceedings of the first ACM international workshop on Mobile entity localization and tracking in GPS-less environments, pages 123-128. ACM, 2008.

[7] G. Zanca, F. Zorzi, A. Zanella, and M. Zorzi. Experimental comparison of RSSI-based localization algorithms for indoor wireless sensor networks. In in REALWSN'08.

[8] K. Srinivasan and P. Levis. Rssi is under appreciated. In Proceedings of EmNets'06, 2006.

[9] D. Lymberopoulos, Q. Lindsey, and A. Savvides. An empirical characterization of radio signal strength variability in 3-d ieee 802.15. 4 networks using monopole antennas. Lecture Notes In Computer Science, $3868: 326,2006$

[10] K. Whitehouse, C. Karlof, and D. Culler. A practical evaluation of radio signal strength for ranging-based localization. ACM SIGMOBILE Mobile Computing and Communications Review, 11(1):52, 2007.

[11] M. Helen, J. Latvala, H. Ikonen, and J. Niittylahti. Using calibration in RSSI-based location tracking system. In Proc. of the 5th World Multiconference on Circuits, Systems, Communications \& Computers (CSCC20001), 2001.

[12] C. Alippi and G. Vanini. A RSSI-based and calibrated centralized localization technique for Wireless Sensor Networks. In Proc. IEEE Int. Conference on Pervasive Computing and Communications Workshops (PERCOMW), pages 301-306, 2006.

[13] N. Patwari and P. Agrawal. Calibration and Measurement of Signal Strength for Sensor Localization. Localization Algorithms and Strategies for Wireless Sensor Networks, page 122, 2009.

[14] A. Awad, T. Frunzke, and F. Dressler. Adaptive distance estimation and localization in wsn using rssi measures. In $D S D$, volume 7, pages 471-478, 2007.

[15] M. Lowton, J. Brown, and J. Finney. Finding NEMO: On the Accuracy of Inferring Location in IEEE 802.15. 4 Networks. In REALWSN'O6, 2006.

[16] F. Cabrera-Mora and J. Xiao. Preprocessing technique to signal strength data of wireless sensor network for real-time distance estimation. In IEEE International Conference on Robotics and Automation, 2008.

[17] K. Nakamura, M. Kamio, T. Watanabe, S. Kobayashi, N. Koshizuka, and K. Sakamura. Reliable ranging technique based on statistical RSSI analyses for an ad-hoc proximity detection system. In Proceedings of the IEEE International Conference on Pervasive Computing and Communications-Volume 00, 2009.

[18] Y. Bar-Shalom, X.R. Li, and T. Kirubarajan. Estimation with applications to tracking and navigation. Wiley-Interscience, 2001.

[19] F. Gustafsson, F. Gunnarsson, N. Bergman, U. Forssell, J. Jansson, R. Karlsson, and P.J. Nordlund. Particle filters for positioning, navigation, and tracking. IEEE Transactions on signal processing, 2002.

[20] G. Mao, B. Fidan, and B.D.O. Anderson. Wireless sensor network localization techniques. Computer Networks, 51(10):2529-2553, 2007.

[21] C. Papamanthou, F.P. Preparata, and R. Tamassia. Algorithms for Location Estimation Based on RSSI Sampling. In Algorithmic Aspects of Wireless Sensor Networks, page 86. Springer, 2008.

[22] AS Chipcon. CC2420 2.4 GHz IEEE 802.15. 4/ZigBee-ready RF Transceiver. Chipcon AS, Oslo, Norway, 4, 2004.

[23] D. Moore, J. Leonard, D. Rus, and S. Teller. Robust distributed network localization with noisy range measurements. In Proceedings of the 2 nd international conference on Embedded networked sensor systems, pages 50-61. ACM New York, NY, USA, 2004.

[24] W.H. Liao and Y.C. Lee. A lightweight localization scheme in wireless sensor networks. In ICWMC'06, 2006.

[25] H.L. Van Trees. Detection, estimation, and modulation theory. WileyInterscience, 2001.

[26] G.D. Forney. The viterbi algorithm. proc. IEEE, 61(3):268-278, 1973.

[27] J.W. Tukey. Exploratory data analysis. 1977. 